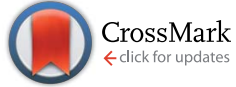

Cite this: Soft Matter, 2014, 10, 8254

\title{
Thermodynamic and kinetic properties of interpolymer complexes assessed by isothermal titration calorimetry and surface plasmon resonance $\dagger$
}

\begin{abstract}
Samuel C. Bizley, Adrian C. Williams and Vitaliy V. Khutoryanskiy*
Interpolymer complexes (IPCs) formed between complimentary polymers in solution have shown a wide range of applications from drug delivery to biosensors. This work describes the combined use of isothermal titration calorimetry and surface plasmon resonance to investigate the thermodynamic and kinetic processes during hydrogen-bonded interpolymer complexation. Varied polymers that are commonly used in layer-by-layer coatings and pharmaceutical preparations were selected to span a range of chemical functionalities including some known IPCs previously characterized by other techniques, and other polymer combinations with unknown outcomes. This work is the first to comprehensively detail the thermodynamic and kinetic data of hydrogen bonded IPCs, aiding understanding and detailed characterization of the complexes. The applicability of the two techniques in determining thermodynamic, gravimetric and kinetic properties of IPCs is considered.
\end{abstract}

Received 26th May 2014

Accepted 27th August 2014

DOI: $10.1039 / c 4 s m 01138 d$

www.rsc.org/softmatter

complexation, whereas a negative enthalpy (exothermic

\section{Introduction}

Interpolymer complexes (IPCs) are formed by mixing two or more complimentary polymers in solutions. A vast range of materials including cationic/anionic polymers, non-ionic systems, smaller molecules (e.g. tannins) and many others can be utilized to produce these complexes, taking advantage of electrostatic attraction, hydrogen bonding, hydrophobic effects and Van der Waals forces; this elicits a large range of complexes whose properties depend on the physical and chemical attributes of the components and on the nature of their interactions. ${ }^{1-7}$

Interpolymer complexes have been used to generate multilayered materials, first developed by Decher, who produced multilayered polyelectrolyte materials on solid substrates. ${ }^{1}$ Subsequently, these products have gained considerable attention due to their ease of production and versatility, and have shown potential applications in drug delivery, biomedicine, electronics, sensors and membrane technologies., ${ }^{2,8-12}$

The ability of polymers to form complexes in solutions is governed by the Gibbs free energy, enthalpy and entropy of the system (see ESI $\dagger$ ). ${ }^{\mathbf{1 5 , 1 6}}$ A positive enthalpy (endothermic process) implies that hydrophobic effects play a substantial role in the

Reading School of Pharmacy, University of Reading, Whiteknights, PO Box 224, Reading, Berkshire, RG6 6AD, UK. E-mail: v.khutoryanskiy@reading.ac.uk; Tel: +44(0) 1183736119

$\dagger$ Electronic supplementary information (ESI) available: Biacore sensorgrams, ITC isotherms and turbidimetric data for all polymer combinations. See DOI: 10.1039/c4sm01138d process) indicates an interaction based on primarily hydrogen bonding. Solution parameters such as $\mathrm{pH}$, ionic strength, temperature and polymer molecular weight have a large impact on the type and strength of interactions. ${ }^{17}$

Interactions between poly(carboxylic acids) and non-ionic polymers result in hydrogen-bonded IPCs. Perez-Gramatges et $a l .{ }^{18}$ and Abe et al. ${ }^{19}$ have shown that the interaction between poly(carboxylic acids) and poly(vinyl pyrrolidone) produces a positive enthalpy change, illustrating the importance of hydrophobic effects in their complexation in addition to hydrogen bonding. Kabanov et $a .^{20}$ studied complexation between poly(ethylene oxide) and poly(methacrylic acid) using potentiometric titration and established that the complexes are stabilized by hydrogen bonding but that other effects such as hydrophobic and conformational interactions resulted in an overall positive enthalpy change. ${ }^{21-24}$ Studies of polyacrylamide with acidic polymers by Staikos et al..$^{25,26}$ have so far been the only polymer-polymer complex to show a negative enthalpic change upon complexation. This is explained by the predominance of hydrogen bonding over other intermolecular interactions; they found that the complexes were relatively weak and easily dissociated with increasing temperature. ${ }^{27,28}$ These studies into hydrogen-bonded IPCs employed flow/conduction calorimetry, or potentiometry to measure thermodynamic parameters.

Isothermal titration calorimetry is a quantitative biochemical tool typically used for characterising protein-ligand, protein-protein and drug-DNA intermolecular interactions. It 
uses stepwise injections of one reagent into another within a calorimetric cell, measuring the heat of the reaction for both exothermic and endothermic processes. ${ }^{\mathbf{1 3 , 1 4}}$ Recently our group demonstrated that isothermal titration calorimetry is a valuable tool in studying complexation between poly(acrylic acid) and methylcellulose. $^{29}$

Surface Plasmon Resonance (SPR) is a powerful and highly sensitive tool used to investigate biomolecular interactions in real time by optically detecting changes in mass on the surface of a sensor chip. It is most widely used to quantify drug-protein binding, antibody characterization, immunogenicity testing and vaccine development. ${ }^{30,31}$ It has been used in tandem with both ellipsometry and quartz crystal micro-balance (QCM) to analyse layer-by-layer deposited polymers. ${ }^{32}$ SPR differs from these aforementioned techniques as it optically detects changes in mass on a surface rather than directly measuring weight change or dielectric properties. Advantages of the technique are that light does not pass through the sample so it can follow coloured or turbid reactions. This technique is thus ideal to study IPC formation in solutions as they are generally opaque. ${ }^{32-34}$ SPR detects not only the mass of polymers deposited on the chip surface, but also provides kinetic parameters including binding affinities and dissociation constants. ${ }^{30,31}$

The kinetics of interpolymer complexation have been sparsely reported and only via stopped-flow and fluorometric methods. ${ }^{35-38}$ Morawetz et al. reported rapid (0.1-0.5 $\left.\mathrm{s}^{-1}\right)$ complexation between poly(acrylic acid) and poly(ethylene oxide) by stopped flow and dynamic tensiometry. ${ }^{35}$ Similar findings were seen for complexes of poly(acrylic acid) with poly(vinyl pyrrolidone)/poly(ethylene glycol) where the initial association between the polymers was very fast $\left(10^{-4} \mathrm{~s}\right)$ but the rate of association was sensitive to both temperature and ionic strength of the solutions.

This study combines Isothermal Titration Calorimetry (ITC) and surface plasmon resonance (Biacore) to elicit fundamental insights into polymer-polymer interactions. Complexation of three acidic materials, namely poly(acrylic acid), poly(methacrylic acid) and tannic acid with 8 non-ionic polymers, namely dextran, 2-hydroxyethyl cellulose, poly(vinyl alcohol), poly(ethylene oxide), poly(vinyl pyrrolidone), polyacrylamide, methylcellulose and poly(methyl vinyl ether) produced 24 potential combinations through which the rate and extent of complexation can be related to polymer structure and their molecular associations.

\section{Experimental section}

\section{Materials}

Poly(acrylic acid) (PAA, $M_{\mathrm{w}} 450 \mathrm{kDa}$ ), tannic acid (TA, $M_{\mathrm{w}} 1701$ Da), dextran (DEX from Leuconostoc spp., $M_{\mathrm{w}} 2000 \mathrm{kDa}$ ), 2-hydroxyethylcellulose (HEC, $M_{\mathrm{w}} 90 \mathrm{kDa}$ ), polyacrylamide (PAM, $\left.M_{\mathrm{w}} 200 \mathrm{kDa}, M_{\mathrm{w}} / M_{\mathrm{n}} 1.1\right)$, poly(ethylene oxide) (PEO, $M_{\mathrm{w}}$ $100 \mathrm{kDa}$ ), methylcellulose (Methocel 60HG or MC, $93 \mathrm{kDa}$ ) with 28-30\% methoxyl content, poly(vinyl pyrrolidone) (PVP, $M_{\mathrm{w}}$ $40 \mathrm{kDa}, M_{\mathrm{w}} / M_{\mathrm{n}} 1.08$ ), poly(methyl vinyl ether) (PMVE, $M_{\mathrm{w}} 20$ $\left.\mathrm{kDa}, M_{\mathrm{w}} / M_{\mathrm{n}} 1.06\right) 50 \% \mathrm{w} / \mathrm{v}$ in water, poly(vinyl alcohol) (PVA, $M_{\mathrm{w}}$ 146-186 kDa, $\left.M_{\mathrm{w}} / M_{\mathrm{n}} 1.4\right)$, were purchased from Sigma-Aldrich
(UK). Poly(methacrylic acid) (PMAA, $M_{\mathrm{w}} 100 \mathrm{kDa}$ ) was purchased from Polysciences Europe. All other chemicals including hydrochloric acid, sodium hydroxide, acetone, phosphate buffer solutions and sodium chloride were purchased from Sigma-Aldrich (UK).

\section{Preparation of polymer solutions}

For SPR analysis, all polymers except PVA were dissolved in deionized water and stirred for a minimum of 8 hours at room temperature to give $0.2 \% \mathrm{w} / \mathrm{v}$ solutions. To prepare the PVA solution, the required amount was firstly dispersed in cold deionized water, then heated to $80{ }^{\circ} \mathrm{C}$ for 30 minutes until dissolved and then cooled back to room temperature ready for use. All solutions were adjusted to $\mathrm{pH} 2.0$ by adding small quantities of $0.1 \mathrm{M} \mathrm{HCl}$ or $\mathrm{NaOH}$ as necessary, and were measured using a digital pH meter (Metrohm, Switzerland). For ITC analysis, all polymers were dissolved in UHQ water for a minimum of 8 hours at room temperature to give either $0.2 \% \mathrm{w} / \mathrm{v}$ solutions for the 8 non-ionic polymers and $2 \% \mathrm{w} / \mathrm{v}$ solutions for the acidic polymer solutions. All solutions were filtered through a $0.4 \mu \mathrm{m}$ filter (Whatman) and degassed by sonication for $30 \mathrm{~min}$.

\section{Isothermal titration calorimetry (ITC)}

ITC analysis used a MicroCal ITC 200 microcalorimeter. Throughout the experiments, $0.2 \% \mathrm{w} / \mathrm{v}$ aqueous solution of each non-ionic polymer was titrated with $2.0 \% \mathrm{w} / \mathrm{v}$ solution of each of the acidic polymer. All experiments were carried out at $25{ }^{\circ} \mathrm{C}$ under continuous stirring of the sample cell at $1000 \mathrm{rpm}$. The titration experiment consisted of 16 injections $(4 \mu \mathrm{L})$ of alternate polymer solutions (beginning with the acidic polymer) at $150 \mathrm{~s}$ intervals. The concentration of polymer was expressed as the mass of the repeat unit (unit $\mathrm{mol} \mathrm{L}^{-1}$ ) calculated from the chemical structure: in the case of MC the molecular weight was calculated allowing for the $28-30 \%$ methoxy content. All calorimetric measurements were performed in triplicate.

\section{Surface plasmon resonance (SPR)}

SPR analysis was performed in a Biacore 3000 equipped with BIA evaluation software using a CM5 sensor chip (GE Healthcare UK). Firstly, a stable baseline with phosphate buffer $(\mathrm{pH}$ 2.0) was established over the course of $10 \mathrm{~min}$. The 24 polymer combinations ( 3 acidic polymers with each of 8 non-ionic polymers) were analysed; each polymer in the combination was alternately applied (4 in total) and SPR sensorgrams were recorded. All experiments were performed at $25{ }^{\circ} \mathrm{C}$ with a polymer injection time of $2 \mathrm{~min}$. After each complete experimental cycle, the cell was injected with $500 \mathrm{mM} \mathrm{NaOH}$ to remove the bound polymer complexes and then flushed with buffer to regenerate the chip surface allowing multiple uses. All Biacore samples were run in triplicate and data was normalized by subtracting the baseline value.

\section{Curve fitting}

Binding affinities and rate constants were determined by local fitting of each of the combinations to an appropriate 1:1 
kinetics model for a general reaction scheme $\mathrm{A}+\mathrm{B}=\mathrm{AB}$ using BIA evaluation 4.1 software. The software calculates the parameters based on a chosen association period between the injection start and $R_{\max }$ values monitoring polymer concentration against time (see Fig. S1, ESI $\dagger$ for graphical details of fitting).

\section{Results and discussion}

\section{Thermal events in the complexation of acidic compounds with non-ionic polymers}

The formation of hydrogen-bonded IPCs in aqueous solutions is known to be strongly dependent on the $\mathrm{pH}$ of polymer solutions. Each interacting pair of polymers is characterised by a critical pH of complexation, above which the formation of IPC is not detected. ${ }^{6}$ For example, previously we demonstrated that the interactions between PAA and MC could not be detected by ITC at $\mathrm{pH}>2.7 .^{29}$ In the present study, in order to establish the effects of polymer nature on complexation and to provide a comparative data for different systems forming IPCs, all polymer solutions were prepared at $\mathrm{pH}$ 2.0. The impact of this is to reduce ionic repulsion between the polymers and to increase the potential for hydrogen bonding.

ITC directly measures the heat change generated by a chemical reaction, which can be exothermic or endothermic depending on the nature of the event. Binding isotherms were generated for the 24 polymer combinations and binding affinities $(K)$ and enthalpies $(\Delta H)$ calculated. $0.2 \% \mathrm{w} / \mathrm{v}$ of each of the 8 non-ionic polymers was titrated against $2 \% \mathrm{w} / \mathrm{v}$ of acidic polymer. Control experiments titrated water against each polymer solution (see Fig. S2 and S3, ESI $\dagger$ ); all isotherms were baseline adjusted accordingly and thus isotherms from polymer mixtures were attributed to complexation and not to polymer dilution.

Fig. 1 shows the isotherms for a selection of polymer combinations (full data Table 1), (A) and (B) use the same nonionic polymer (MC) but different acidic substrates (PAA and PMAA, respectively). The presence of binding isotherms confirms that complexation occurred for both sets of polymers and results are comparable to our previous findings $(0.59 \mathrm{~kJ}$ base-mol $^{-1}$ for PAA-MC). ${ }^{29}$ The isotherms show very similar results both qualitatively and quantitatively. Previous reports have shown that, under the correct conditions (in terms of $\mathrm{pH}$ and ionic strength), PMAA shows increased hydrophobic effects compared to PAA resulting in a larger binding isotherm but under the conditions employed here no statistical difference in terms of binding affinity $(K)$ and enthalpy $(H)$ between samples was seen. ${ }^{19,39,40}$ Interaction between tannic acid (TA) and MC showed a considerably higher enthalpy change and binding affinity (Table 1), illustrating increased complexation compared to PAA. Recently we reported greater $\mathrm{pH}$ stability of TA-MC complexes compared to PAA-MC. ${ }^{\mathbf{1 1}}$

Each of the acidic compounds showed no detectable thermal events in combination with dextran demonstrating that no complexation occurred. This was expected since dextran is a highly hydrophilic polysaccharide and so binds strongly with water which cannot be displaced by the addition of a polymer.
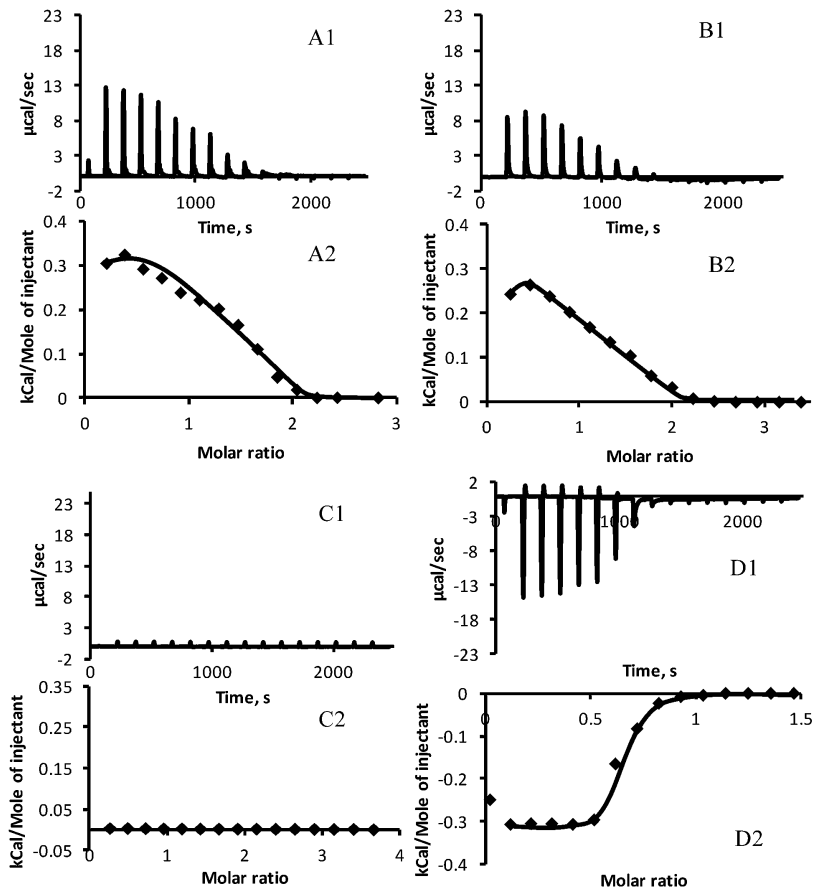

Fig. 1 Isothermal titration calorimetry isotherms obtained by titrating (A1 + A2) $0.2 \% \mathrm{w} / \mathrm{v}$ methylcellulose with $2 \% \mathrm{w} / \mathrm{v}$ poly(acrylic acid), (B1 + B2) $0.2 \%$ methylcellulose with $2 \% \mathrm{w} / \mathrm{v}$ poly(methacrylic acid), (C1 + C2) $0.2 \% \mathrm{w} / \mathrm{v}$ dextran with $2 \% \mathrm{w} / \mathrm{v}$ poly(acrylic acid), (D1 + D2) $0.2 \% \mathrm{w} / \mathrm{v}$ polyacrylamide with $2 \% \mathrm{w} / \mathrm{v}$ poly(acrylic acid) solutions all at $\mathrm{pH} 2.0 .1$ denotes raw graphical data which has been transformed to measure the relationship of concentration labelled 2 whose fitting allows parameter determination. (see Fig. S4-S6, ESI† for the full set of ITC isotherms for all combinations.)

This result was confirmed with a turbidimetric study (see Fig. S7, ESI $\dagger$ for exemplar images) whereby the two solutions were mixed and no change in turbidity was recorded, in contrast to all other combinations where turbidity changed confirming the formation of a complex even for samples that showed very low enthalpy changes (PAA-HEC did show a turbidity change though a full isotherm could not be recorded due to the low level of binding).

The majority of combinations produced endothermic reactions except for complexes between TA and PVP or HEC, and all PAM combinations gave exothermic interactions emphasizing that different intermolecular forces can contribute to IPC formation. For the endothermic complexations, variations in enthalpic magnitude between combinations illustrate that though the nature of the interaction is the same, the affinities differ.

Structurally similar polymers show some interesting differences in complexation behaviour. Both HEC and MC are derivatives of cellulose and only vary in the nature of the substituent groups (hydroxyethyl and methyl for HEC and MC, respectively), but each HEC combination showed consistently lower binding affinity and enthalpy change compared with the respective MC complexes. These results demonstrate that complexation is sensitive to relatively small changes in functional groups, such as an increase in length or hydrophobicity 
Table 1 ITC analysis of stoichiometry $(n)$, binding affinity $(K)$ and change in enthalpy for each polymer combination, $0.2 \%$ w/v non-ionic polymer was titrated into $2 \% \mathrm{w} / \mathrm{v}$ acidic compound. Data shown as mean \pm standard deviation. a and b denote statistical significance $(p<0.05)$ within each group of polymer combinations, calculated using ANOVA and Bonferroni post-hoc tests on Prism (Graphpad, USA). Different letter indicates that the difference is statistically significant

\begin{tabular}{|c|c|c|c|}
\hline Polymer combination & $N$ & $K$, base-mol ${ }^{-1}\left(\times 10^{3}\right)$ & $\Delta H, \mathrm{~kJ}$ base-mol ${ }^{-1}$ \\
\hline PAA-DEX & 0 & 0 & 0 \\
\hline PMAA-DEX & 0 & 0 & 0 \\
\hline TA-DEX & 0 & 0 & 0 \\
\hline PAA-HEC & 0 & 0 & $0.03 \pm 0.02$ \\
\hline PMAA-HEC & 4.25 & $0.550 \pm 0.045^{\mathrm{a}}$ & $0.17 \pm 0.07$ \\
\hline TA-HEC & 0.27 & $8.260 \pm 0.871^{\mathrm{b}}$ & $-3.25 \pm 0.21^{\mathrm{a}}$ \\
\hline PAA-PVA & 0.59 & $0.125 \pm 0.010$ & $0.03 \pm 0.01$ \\
\hline PMAA-PVA & 0.53 & $7.080 \pm 0.246^{\mathrm{a}}$ & $0.15 \pm 0.03^{\mathrm{a}}$ \\
\hline TA-PVA & 0.062 & $6.010 \pm 0.194^{\mathrm{b}}$ & $2.21 \pm 0.25^{\mathrm{b}}$ \\
\hline PAA-MC & 1.32 & $2.300 \pm 0.167$ & $0.64 \pm 0.04$ \\
\hline PMAA-MC & 1.40 & $2.090 \pm 0.211$ & $0.51 \pm 0.06$ \\
\hline TA-MC & 0.06 & $11.500 \pm 0.432^{\mathrm{a}}$ & $2.25 \pm 0.27^{\mathrm{a}}$ \\
\hline PAA-PEO & 0.67 & $3.370 \pm 0.312$ & $0.57 \pm 0.04$ \\
\hline PMAA-PEO & 0.68 & $4.430 \pm 0.341$ & $0.67 \pm 0.07$ \\
\hline TA-PEO & 0.03 & $1.390 \pm 0.564^{\mathrm{a}}$ & $2.95 \pm 0.19^{\mathrm{a}}$ \\
\hline PAA-PVP & 0.42 & $3.650 \pm 0.213$ & $0.31 \pm 0.06$ \\
\hline PMAA-PVP & 1.01 & $3.030 \pm 0.412$ & $0.35 \pm 0.07$ \\
\hline TA-PVP & 0.001 & $2.240 \pm 0.116^{\mathrm{a}}$ & $-0.67 \pm 0.10^{\mathrm{a}}$ \\
\hline PAA-PAM & 0.57 & $7.900 \pm 0.854$ & $-1.20 \pm 0.07$ \\
\hline PMAA-PAM & 0.67 & $3.740 \pm 0.351$ & $-1.06 \pm 0.09$ \\
\hline TA-PAM & 0.06 & $22.100 \pm 3.214^{\mathrm{a}}$ & $-8.25 \pm 0.99^{\mathrm{a}}$ \\
\hline PAA-PMVE & 1.44 & $12.500 \pm 1.983$ & $1.54 \pm 0.32$ \\
\hline PMAA-PMVE & 1.49 & $10.100 \pm 0.974$ & $1.92 \pm 0.26$ \\
\hline TA-PMVE & 0.07 & $206.000 \pm 9.742^{\mathrm{a}}$ & $12.44 \pm 0.67^{\mathrm{a}}$ \\
\hline
\end{tabular}

of side chains; Nurkeeva et al. ${ }^{43}$ reported higher critical $\mathrm{pH}$ values for IPCs formed by MC and PMAA compared to PMAAHEC complexes. This difference was attributed to the existence of a lower critical solution temperature (LCST) in MC solutions and the absence of this property in HEC. LCST in aqueous solutions indicates the higher contribution of hydrophobic effects on polymer conformation and on stabilisation of their complexes with poly(carboxylic acids). ${ }^{44}$ Interestingly, TA gave different thermal effects when complexed with HEC or MC with a negative enthalpy change for HEC and positive for MC. Whilst the binding affinity is very similar for the two, the enthalpic discrepancies show that the nature of the association is quite different; conformational differences affecting TA's proximity to the polymer backbone is one potential explanation for this due to the increased side chain length of HEC, though this result was not mirrored for PAA and PMAA with HEC or MC. Complexes of PAA or PMAA with PEO (100 kDa) gave binding enthalpies of $0.57 \pm 0.04$ and $0.67 \pm 0.07 \mathrm{~kJ} \mathrm{base-mol}^{-1}$, respectively. Eagland et $a .^{42}$ found that the enthalpy of the PMAA-PEO (35 kDa) complex measured by flow calorimetry was $0.35 \mathrm{~kJ}$ base- $\mathrm{mol}^{-1}$. This discrepancy can be attributed to the different molecular weight of the PEO used in our study, as higher molecular weight polymers are known to form stronger IPCs. $^{42}$

The combination of each of the acidic compounds with PMVE showed the strongest thermal events $(\Delta H 1.54,1.92$ and $12.44 \mathrm{~kJ}$ base-mol ${ }^{-1}$ for PAA, PMAA and TA, respectively) and high binding affinities showed that there is strong complexation upon mixing of the two polymers in aqueous solutions. The isotherms gave positive enthalpies demonstrating that hydrophobic effects contribute to the stabilisation of IPCs.

PAM combinations consistently gave negative binding isotherms with all of the acidic compounds with negative enthalpy changes. These results agree with Staikos et $a .^{25}$ who potentiometrically recorded a negative enthalpy change for PAA-PAM complexation, though the magnitude of their enthalpy was much higher than recorded here by ITC $(-75 \mathrm{~kJ}$ $\mathrm{mol}^{-1}$ compared with our $-1.20 \mathrm{~kJ}$ base- $\mathrm{mol}^{-1}$ ); this can be attributed to differences in solution $\mathrm{pH}$ and concentration as this system is very responsive to changes in $\mathrm{pH}$, ionic strength and temperature. The negative enthalpies demonstrate that this system is predominantly stabilized by hydrogen bonding, which correlates well with the conclusions reported by Koussathana et al. ${ }^{\mathbf{4 4}}$ They reported that PAA-PAM complexes do not show any 
stabilization via hydrophobic effects and related this to the structural features and properties of PAM such as the upper critical solution temperature (UCST) as well as the hydrophilic nature of its amide groups.

In most cases PAA and PMAA show broadly similar isotherm patterns, magnitudes of binding, enthalpy changes and binding affinities except for HEC and PVA. The position of carboxylic groups is the same in both PAA and PMAA and so in principle they are chemically similar. The presence of the methyl group in PMAA may have some effect on the conformation of its macromolecules and stabilization of the IPC via hydrophobic effects which may account for the differences seen for HEC and PVA. In contrast, TA showed significantly different ITC results, as it has a very different chemical structure to PAA and PMAA, both in terms of the nature of its acidic groups (phenolic groups in TA compared to carboxylic groups in PAA and PMAA) and molecular weight.

Clearly, ITC is a powerful tool that can be used to study a broad range of systems, such as complexes formed by oppositely charged polyelectrolytes.

\section{SPR analysis of the complexation of acidic compounds with non-ionic polymers}

Surface plasmon resonance does not analyse the thermal events occurring when the polymers are mixed but measures optically the change in mass on the sensor chip surface. However, we expected the increase in mass on the surface to correlate well with the enthalpy measured by ITC, as the greater mass deposited should result from increased binding with consequent changes in enthalpy.

Fig. 2 shows exemplar sensorgrams for combinations of each acidic compound with dextran, polyacrylamide or methylcellulose. The initial injection of acidic compound saturates the sensor chip surface, followed by injection of the complimentary non-ionic polymer. A total of 4 injections were carried out for each sample following a standard, previously optimized
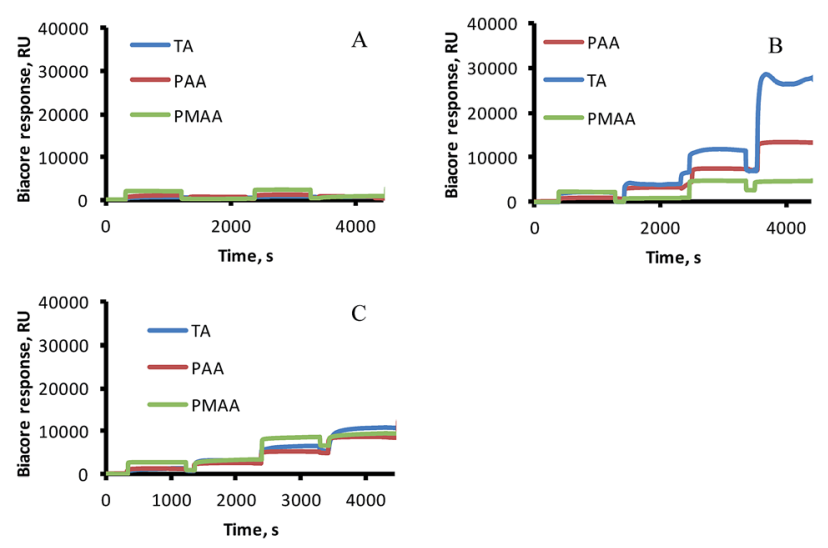

Fig. 2 Biacore sensorgrams for the formation of IPCs between acidic compounds and DEX (A), PAM (B), and MC (C). Concentrations are $0.2 \% \mathrm{w} / \mathrm{v}$ for acidic compound and $0.2 \% \mathrm{w} / \mathrm{v}$ for non-ionic polymer solutions at $\mathrm{pH}$ 2.0. (see Fig. S8, ESI† for the full set of Biacore sensorgrams for all combinations).

layer-by-layer cycle, ${ }^{33}$ with data selected from the interaction between layers 3 and 4 .

Combinations of the acidic compounds with DEX showed only signals associated with the initial deposition of the acidic material, confirming that no subsequent complexation occurs, in agreement with the above ITC data and with Nurkeeva et al. ${ }^{\mathbf{4 3}}$ who found no phase separation in solutions of PAA and DEX over a wide range of $\mathrm{pHs}$. This sample also acted as an internal control to verify that subsequent addition of acidic compounds to the sensor chip did not result in an increase in signal. This confirms firstly that the proton-donating material does not preferentially complex with itself and secondly that the sensor chip is saturated with the polymer, as any unbound available carboxylated dextran would be complexed with the further polymer addition, and so an increased signal would occur which is not seen here.

The greatest degree of complexation was again seen for combinations with PMVE that showed an $R_{\max }$ value of up to $23800 \mathrm{RU}$ (TA-PMVE), in agreement with the data from the ITC.

Table 2 SPR analysis of binding association $\left(k_{\mathrm{a}}\right)$ and maximal Biacore response $\left(R_{\max }\right)$ for each polymer combination. $0.2 \% \mathrm{w} / \mathrm{v}$ acidic compound was alternately layered with $0.2 \% \mathrm{w} / \mathrm{v}$ non-ionic polymer for a total of 4 additions. Data shown as mean \pm standard deviation from triplicate runs. $\mathrm{a}$ and $\mathrm{b}$ denote statistical significance $(p<0.05)$ within each group of polymer combinations, calculated using ANOVA and Bonferroni post-hoc tests on Prism (Graphpad, USA). Different letter indicates that the difference is statistically significant

Polymer

combination

$k_{\mathrm{a}}, \mathrm{M}^{-1} \mathrm{~s}^{-1}$

PAA-DEX

PMAA-DEX

TA-DEX

PAA-HEC

PMAA-HEC

TA-HEC

PAA-PVA

PMAA-PVA

TA-PVA

PAA-PEO

PMAA-PEO

TA-PEO

PAA-MC

PMAA-MC

TA-MC

PAA-PVP

PMAA-PVP

TA-PVP

PAA-PAM

PMAA-PAM

TA-PAM

PAA-PMVE

PMAA-PMVE

TA-PMVE
0
0

$0.406+0.048$

$0.368 \pm 0.013$

$0.120 \pm 0.018^{\mathrm{a}}$

$0.828 \pm 0.160$

$0.778 \pm 0.048$

$0.651 \pm 0.049$

$1.310 \pm 0.270$

$1.180 \pm 0.240$

$0.651 \pm 0.130^{\mathrm{a}}$

$3.170 \pm 0.830$

$2.720 \pm 0.640$

$0.812 \pm 0.040^{\mathrm{a}}$

$3.240 \pm 0.450$

$2.600 \pm 0.390$

$0.000^{\mathrm{a}}$

$4.260 \pm 0.610$

$4.190 \pm 0.410$

$1.260 \pm 0.578^{\mathrm{a}}$

$11.500 \pm 1.720$

$10.700 \pm 0.820$

$1.770 \pm 0.015^{\mathrm{a}}$
$R_{\max }, \mathrm{RU}$

0

0

0

$463 \pm 57$

$1898 \pm 173^{\mathrm{a}}$

$15800 \pm 1132^{\mathrm{b}}$

$2960 \pm 284^{\mathrm{a}}$

$5187 \pm 590$

$4851 \pm 673$

$3863 \pm 231$

$3000 \pm 468$

$3850 \pm 514$

$5988 \pm 753^{\mathrm{ab}}$

$4851 \pm 431^{\mathrm{a}}$

$6722 \pm 597^{\mathrm{b}}$

$6390 \pm 653$

$5960 \pm 435$

$895 \pm 102^{\mathrm{a}}$

$9960 \pm 764$

$6014 \pm 731^{\mathrm{a}}$

$19500 \pm 1586^{\mathrm{b}}$

$14200 \pm 1401$

$14100 \pm 965$

$23800 \pm 2865^{\mathrm{a}}$ 
Comparing PAA and PMAA complexes with the non-ionic polymers, the Biacore data shows few statistical differences between complexes. PAA and PMAA with HEC correlated with the differences in ITC data which was attributed to differences in hydrophobic effects enhanced by the methyl groups of the PMAA. PAA and PMAA with PAM also showed marked differences, the ITC confirmed that hydrogen bonding was the overriding interaction and the additional methyl groups on the PMAA are not very efficient in providing additional stabilization of IPCs by hydrophobic effects.

Interestingly, Biacore also allowed kinetic information to be extracted from the data, giving both association and dissociation rates for the polymers. The data (Table 2) shows that for all combinations, TA complexes form slower than those with the poly(carboxylic acids) which complex rapidly. For example, most rapid complexes form with PMVE where for TA the binding association is $1.77 \mathrm{M}^{-1} \mathrm{~s}^{-1}$ but for PAA with PMVE is
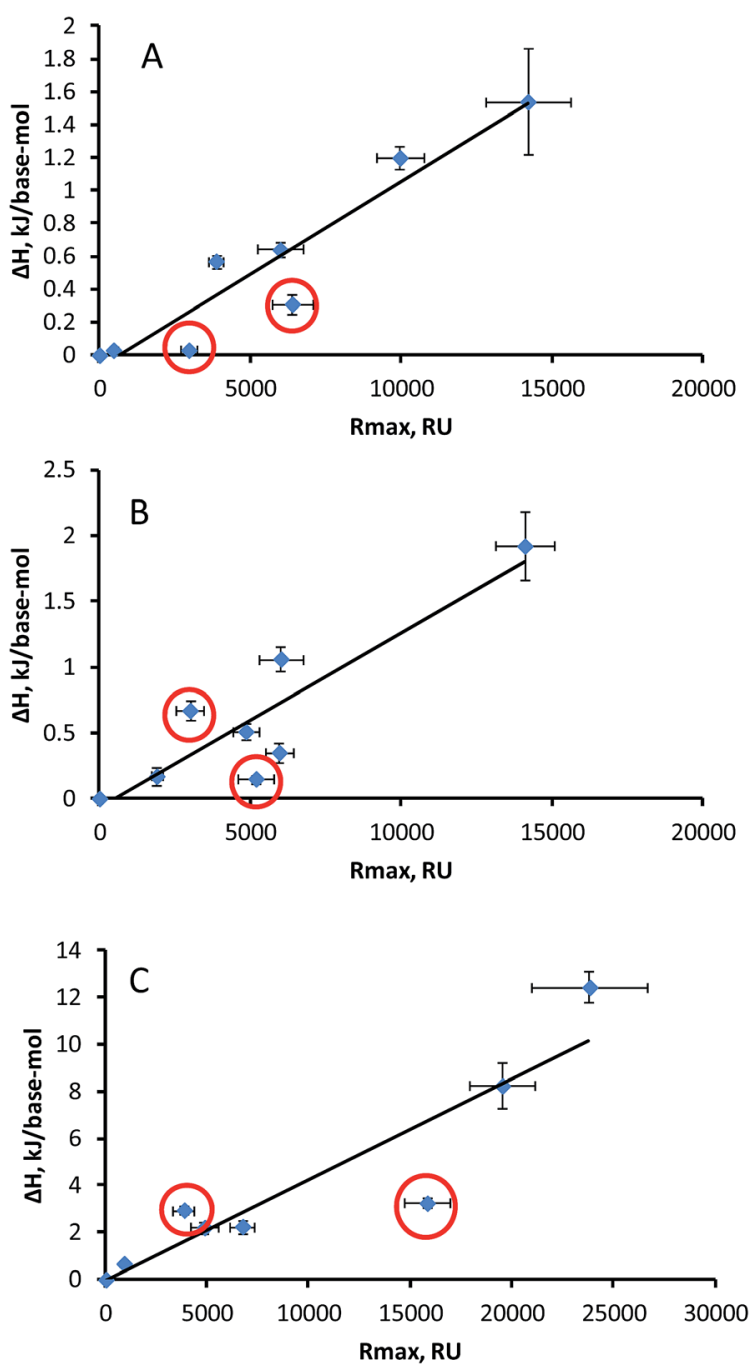

Fig. 3 Correlation between enthalpy $(\Delta H)$ and Biacore response for IPCs formed by PAA (A), PMAA (B) and TA (C) with each of the nonionic polymers, outliers (red) were not included in the linear fitting, and were defined as outside 3 standard deviations using Grubbs' test (98\% confidence level). over 6-fold quicker at $11.5 \mathrm{M}^{-1} \mathrm{~s}^{-1}$. The decreased rate of complexation with TA does not necessarily mean that the overall complexation strength is lower though, as TA shows the highest $R_{\max }$ results for all combinations so the degree of complexation was greater. This discrepancy appears counterintuitive but is explained by the stoichiometry and enthalpy data obtained by ITC (Table 1) which showed that the number of binding sites was markedly higher and the enthalpy per basemol was 6 to 8-fold greater for the TA samples with PMVE compared with the poly(carboxylic acids). Thus, though the rate of association is slower, the extent of binding is greater resulting in larger Biacore signals.

Morawetz et $a{ }^{24}{ }^{24}$ measured the kinetics of PAA interacting with both PVP and PEO using fluorescence emission decay; the rate of complexation was found to be strongly dependent on salt concentration and molecular weight with PAA-PVP complexation occurring in the order of 0.0038-0.011 $\mathrm{s}^{-1}$, and complexation between PAA and PEO occurred with a reduced complexation rate $\left(0.00018-0.0096 \mathrm{~s}^{-1}\right)$. Direct comparison cannot be made with our results as Biacore measures kinetics as a function of concentration (hence the different units), in contrast to the fluorescence emission decay technique. Biacore, however, does show that the binding rate for PAA-PVP (3.24 \pm $\left.0.45 \mathrm{M}^{-1} \mathrm{~s}^{-1}\right)$ is significantly higher than for PAA-PEO $(1.31 \pm$ $\left.0.27 \mathrm{M}^{-1} \mathrm{~s}^{-1}\right)$, in agreement with the results of Morawetz et al. ${ }^{24}$

Due to the significantly slower association rate but greater degree of complexation seen for TA, data from this acidic compound cannot be directly compared to the two poly(carboxylic acids). However, it is noteworthy that complexes show the same trend as seen from the ITC data with DEX and HEC giving the lowest binding association (e.g. zero and 0.406 $\mathrm{M}^{-1} \mathrm{~s}^{-1}$ for PAA complexes, respectively) and PAM and PMVE providing the highest rate of complexation (e.g. 4.26 and 11.5 $\mathrm{M}^{-1} \mathrm{~s}^{-1}$ for PAA complexes).

A comparison of the thermodynamic parameters of the complexation for different polymeric systems recorded using ITC with the Biacore data gives a clear positive correlation between $\Delta H$ and Biacore response values (Fig. 3). This correlates the mass of IPC deposited on Biacore sensor chip with the heat generated/absorbed during complexation.

\section{Conclusion}

Isothermal titration calorimetry and surface plasmon resonance studies have been used to characterise the rate and extent of complexation for three acidic compounds with 8 non-ionic polymers. The data shows varying degrees of complex formation in an acidic environment, from DEX which is unable to form complexes to PMVE which generates strong and rapid interactions. For the poly(carboxylic acids) (PAA and PMAA), their structural similarity resulted in minor differences in the rate, degree or energetics of complexation. In contrast, tannic acid has significant potential for use as a material in generating robust layer-by-layer structures with strong complexes formed in nearly all combinations, though the rate of formation was relatively slow. 
The two techniques analyse different parameters and together are highly complementary to provide a comprehensive understanding of IPC formation. Whilst the data cannot be directly compared, they give consistent results in terms of rank orders and magnitudes. Both techniques offer relatively rapid throughput with real time analysis, and are highly sensitive. ITC provides binding affinities, enthalpies and from this, the forces operating in complex formation can be interpolated. Surface plasmon resonance provides data on the physical mass of the complex produced and the rate of interactions. Separately they provide valuable but partial understanding of IPC formation but in combination they give a comprehensive insight into the events occurring when two polymers are combined.

\section{Acknowledgements}

We are grateful for the funding and support of the Engineering and Physical Sciences Research Council for Doctorial Training Grant to SB (EP/P505682/1), and would like to acknowledge the Chemical Analysis Facility at the University of Reading for the use of equipment and expertise.

\section{References}

1 G. Decher, Science, 1997, 277, 1232.

2 E. Kharlampieva, V. Kozlovskaya and S. A. Sukhishvili, Adv. Mater., 2009, 21, 3053.

3 W. B. Stockton and M. F. Rubner, Macromolecules, 1997, 30, 2717.

4 L. Wang, Z. Wang, X. Zhang, J. Shen, L. Chi and H. Fuchs, Macromol. Rapid Commun., 1997, 18, 509.

5 F. Caruso, K. Niikura, D. N. Furlong and Y. Okahata, Langmuir, 1997, 13, 3422.

6 Hydrogen-bonded Interpolymer Complexes: Formation, Structure and Applications, ed. V. V. Khutoryanskiy and G. Staikos, World Scientific, 2009, p. 376, ISBN 978-981-270785-7.

7 V. V. Khutoryanskiy, Int. J. Pharm., 2007, 334, 15.

8 A. Antipov and G. B. Sukhorukov, Adv. Colloid Interface Sci., 2004, 111, 49.

9 Y. Lvov, A. A. Antipov, A. Mamedov, H. Möhwald and G. B. Sukhorukov, Nano Lett., 2001, 1, 125.

10 S. B. Kim, S. W. Park and P. T. Hammond, ACS Nano, 2008, 2, 386.

11 K. Ariga, M. McShane, Y. M. Lvov, O. Ji and J. P. Hill, Expert Opin. Drug Delivery, 2011, 8, 633.

12 P. Podsiadlo, L. Sui, Y. Elkasabi, P. Burgardt, J. Lee, A. Miryala, W. Kusumaatmaja, M. R. Carman, M. Shtein, J. Kieffer, J. Lahann and N. A. Kotov, Langmuir, 2007, 23, 7901.

13 J. E. Grolier and J. M. Rio, Int. J. Mol. Sci., 2009, 10, 5296.

14 A. Velazquez, S. A. Leavitt and E. Freire, Characterization of protein-protein interactions by isothermal titration calorimetry, Humma Press Inc., 2007, vol. 261, p. 35.

15 A. Ababou and E. J. Ladbury, J. Mol. Recognit., 2007, 21, 4.
16 M. J. Blandamer, P. M. Cullis and J. B. F. N. Engberts, J. Chem. Soc., Faraday Trans., 1998, 94, 2261.

17 A. Saboury, J. Iran. Chem. Soc., 2006, 3, 1.

18 A. Perez-Gramatges, W. Arguelles-Monal and C. PenicheCovas, Polym. Bull., 1996, 37, 127.

19 K. Abe, H. Ohno, A. Nii and E. Tsuchida, Makromol. Chem., 1978, 179, 2043.

20 V. Y. Baranovski, I. D. Zenkov and V. Kabanov, Vysokomol. Soedin., Ser. A, 1989, 31, 493.

21 V. Y. Baranovsky, A. A. Litmanovich, I. M. Papisov and V. A. Kabanov, Eur. Polym. J., 1981, 17, 969.

22 N. J. Turro, G. Caminati and J. Kim, Macromolecules, 1991, 24, 4054.

23 V. Vilar, L. Irusta, M. J. Fernandez-Berridi, J. J. Iruin, M. Iriarte, L. Gargallo and D. Radic, Thermochim. Acta, 2003, 402, 209.

24 B. Bednar, H. Morawetz and J. A. Shafer, Macromolecules, 1984, 17, 1634.

25 G. Staikos, K. Karayanni and Y. Mylonas, Macromol. Chem. Phys., 1997, 198, 2905.

26 G. Bokias and G. Staikos, Recent Res. Dev. Macromol. Res., 1999, 4, 247.

27 Y. Osada and M. Sato, Polym. Lett., 1976, 14, 129.

28 V. V. Khutoryanskiy, A. Dubolazov, Z. S. Nurkeeva and G. A. Mun, Langmuir, 2004, 9, 3785.

29 O. V. Khutoryanskaya, A. C. Williams and V. V. Khutoryanskiy, Macromolecules, 2007, 40, 7707.

30 S. C. B. Gopinath, Sens. Actuators, B, 2010, 150, 722.

31 X. D. Hoa, A. G. Kirk and M. Tabrizian, Biosens. Bioelectron., 2007, 23, 151.

32 W. R. Nelson and J. R. Krone, J. Mol. Recognit., 1999, 12, 77.

33 S. C. Bizley, A. C. Williams, F. Kemp and V. V. Khutoryanskiy, Soft Matter, 2012, 8, 6782.

34 M. Piliarik, H. Vaisocherova and J. Homola, Methods Mol. Biol., 2009, 503, 65.

35 H. L. Chen and H. Morawetz, Eur. Polym. J., 1983, 19, 923.

36 A. Kleingartner, H. Lee, M. Rubner, G. H. McKinley and R. E. Cohen, Soft Matter, 2013, 9, 6080.

37 J. Zhang, S. Chen, Z. Zhu and S. Liu, Phys. Chem. Chem. Phys., 2014, 16, 117.

38 S. Luo, S. Liu, J. Xu, H. Liu, Z. Zhu, M. Jiang and C. Wu, Macromolecules, 2006, 39, 4517.

39 C. Alkan, E. Gunther, S. Hiebler and M. Himple, Energy Conservation and Management, 2012, 64, 364.

40 I. Iliopoulos and R. Audebert, Macromolecules, 1991, 24, 2566. 41 K. Driver, S. Baco and V. V. Khutoryanskiy, Eur. Polym. J., 2013, 49, 4249.

42 D. Eagland, N. J. Crowther and C. J. Butler, Eur. Polym. J., 1994, 30, 767.

43 Z. S. Nurkeeva, G. A. Mun and V. V. Khutoryanskiy, Macromol. Biosci., 2003, 3, 283.

44 M. Koussathana, P. Lianos and G. Staikos, Macromolecules, 1997, 30, 7798. 